\title{
Development of a patient-centered aggregate score to predict survival after lung resection for non-small cell lung cancer
}

\author{
Alessandro Brunelli, MD, ${ }^{\text {a,b }}$ Michele Salati, MD, ${ }^{a}$ Majed Refai, MD, ${ }^{\text {a }}$ Francesco Xiumé, MD, ${ }^{\text {a }}$ \\ Rossana Berardi, MD, ${ }^{\mathrm{c}}$ Paola Mazzanti, MD, ${ }^{\mathrm{c}}$ and Cecilia Pompili, $\mathrm{MD}^{\mathrm{a}}$
}

Objective: The objective of this analysis was to develop a survival aggregate score (SAS), including objective and subjective patient-based parameters, and assess its prognostic role after major anatomic resection for non-small cell lung cancer.

\begin{abstract}
Methods: A total of 245 patients underwent major lung resections for non-small cell lung cancer with preoperative evaluation of quality of life (Short-Form 36v2 survey) and complete follow-up. The Cox multivariable regression and bootstrap analyses were used to identify prognostic factors of overall servival, which were weighted to construct the scoring system and summed to generate the SAS.

Results: Cox regression analysis showed that the factors negatively associated with overall survival and used to construct the score were 36-item short-form health survey physical component summary score less than 50 (hazard ratio $[\mathrm{HR}], 1.7 ; P=.008)$, aged older than 70 years (HR, 1.9; $P=.002)$, and carbon monoxide lung diffusion capacity less than $70 \%(\mathrm{HR}, 1.7 ; P=.01)$. Patients were grouped into 4 risk classes according to their SAS. The 5 -year overall survival was $78 \%$ in class SAS0, $59 \%$ in class SAS1, $42 \%$ in class SAS2, and $14 \%$ in class SAS3 (log-rank test, $P<.0001)$. SAS maintained its association with overall survival in patients with stages pT1 (log-rank test, $P=.01$ ), pT2 (log-rank test, $P=.02$ ), or pT3-4 (log-rank test, $P=.001$ ), and in those with stages pN0 (log-rank test, $P=.0005$ ) or pN1-2 (log-rank test, $P=.02$ ). The 5 -year cancer-specific survival was $83 \%$ in class SAS0, $71 \%$ in class SAS1, $63 \%$ in class SAS2, and $17 \%$ in class SAS3 (log-rank test, $P<.0001$ ).
\end{abstract}

Conclusions: This system may be used to refine stratification of prognosis for clinical and research purposes.

(J Thorac Cardiovasc Surg 2013;146:385-90)

Supplemental material is available online.

Earn CME credits at

http://cme.ctsnetjournals.org

Objective measures of performance ${ }^{1-3}$ and patientperceived physical status ${ }^{4-6}$ have been associated with survival time after surgery in patients with non-small cell lung cancer (NSCLC).

The objective of this study was to develop an aggregate scoring system, incorporating objective and subjective patient-based parameters, and to assess its association

\footnotetext{
From the Divisions of Thoracic Surgery ${ }^{\mathrm{a}}$ and Clinical Oncology ${ }^{\mathrm{c}}$ and the Section of Minimally Invasive Thoracic Surgery, ${ }^{\mathrm{b}}$ Ospedali Riuniti Ancona, Ancona, Italy. Disclosures: Authors have nothing to disclose with regard to commercial support. Received for publication Jan 7, 2013; revisions received Feb 8, 2013; accepted for publication April 3, 2013; available ahead of print May 6, 2013.

Address for reprints: Alessandro Brunelli, MD, Division of Thoracic Surgery, Ospedali Riuniti Ancona, Via Conca 1, 60122 Ancona, Italy (E-mail: brunellialex@gmail.com).

$0022-5223 / \$ 36.00$

Copyright (c) 2013 by The American Association for Thoracic Surgery http://dx.doi.org/10.1016/j.jtcvs.2013.04.007
}

with prognosis in patients undergoing curative anatomic resection for NSCLC.

\section{METHODS}

This is a prospective longitudinal study on 407 patients who underwent lobectomy or pneumonectomy and systematic nodal dissection for NSCLC from January 2004 to December 2008. The stages of patients were determined according to the American Joint Committee on Cancer guidelines. ${ }^{7}$

A total of 311 patients underwent preoperative assessment of quality of life (QoL). Sixty-six of them were lost to follow-up. The remaining 245 patients (214 lobectomies and 31 pneumonectomies) were analyzed. A preliminary analysis showed that the patients with preoperative QoL assessment (included in the study) had similar overall 5-year survival (55\% vs $56 \%$; log-rank test, $P=.9$ ) compared with those without QoL assessment (excluded from the study).

This study was approved by the hospital Institutional Review Board, and all patients gave their informed consent to use their clinical data for scientific purposes. Operability exclusion criteria included a predicted postoperative forced expiratory volume in 1 second $\left(\mathrm{FEV}_{1}\right)$ and a predicted postoperative DLCO lower than $30 \%$, in addition to an oxygen consumption per unit time peak lower than $10 \mathrm{~mL} / \mathrm{kg}$ per minute. As a rule, operations were performed through a lateral muscle- and nerve-sparing thoracotomy by Board-certified thoracic surgeons.

Patients were extubated in the operating room and transferred to a dedicated thoracic ward. Postoperative management focused on early mobilization, antithrombotic and antibiotic prophylaxis, and physical and respiratory rehabilitation. Thoracotomy chest pain was assessed at least twice daily and controlled through a systemic, continuous infusion of nonopioid drugs. Therapy was titrated to achieve a visual analogue score lower than 5 (scale range, $0-10$ ) during the first 48 to 72 hours. No formal 


Abbreviations and Acronyms
CI $=$ confidence interval
DLCO $=$ carbon monoxide lung diffusion capacity
ECOG $^{=}$Eastern Cooperative Oncology group
FEV $_{1}=$ forced expiratory volume in 1 second
FVC $=$ forced vital capacity
HR $=$ hazard ratio
LTFU $=$ lost to follow-up
MCS $=$ mental component summary
NSCLC $=$ non-small cell lung cancer
PCS $=$ physical component summary
QoL $=$ quality of life
SAS $=$ survival aggregate score
SF36 $=36$-item short-form health survey
SF36v2 $=$ Short-Form 36v2

preadmission or postdischarge physiotherapy or psychological supportive programs were administered.

Neurological or psychotropic personal medications, if present, were generally resumed the day after surgery.

\section{QoL Assessment}

QoL was assessed before the operation by the administration of the Short-Form 36v2 (SF36v2) survey. The SF36v2 questionnaire ${ }^{8}$ is a generic instrument assessing 8 health physical and mental concepts (physical functioning, role limitation caused by physical problems, bodily pain, general health perception, vitality, social functioning, role limitation caused by emotional problems, and mental health). Scores were standardized to norms, and their weighted averages were used to create physical component summary (PCS) and mental component summary (MCS) scores on a standard scale. Norm-based scores have a mean of 50 and an SD of 10. As a consequence, for all health dimensions and component scales, any score lower than 50 is lower than the general population mean and each point represents one tenth of an SD. This allows for a direct comparison of measures among different populations and scales.

\section{Survival}

Follow-up was obtained by routine office visits, telephone contact, or data retrieved from the Regional Health Care System database. All patients were observed through April 2011. The cause of death was recorded based on physician report, ascertaining death for those cases retrieved from the Regional Health Care System database or reported by family members when telephone contact was used. The median follow-up calculated by using the reverse Kaplan-Meier method was 37 months. Perioperative mortality occurred in 3 patients in this series, and these events were counted as overall deaths and included in the survival analysis.

\section{Statistical Analysis}

The following baseline and tumor variables were tested for a possible association with survival: age, sex, body mass index, American Society of Anesthesiologist score, Eastern Cooperative Oncology group (ECOG) score, $\mathrm{FEV}_{1}$ percentage, carbon monoxide lung diffusion capacity (DLCO percentage), $\mathrm{FEV}_{1}$ /forced vital capacity (FVC) ratio, history of coronary artery disease, renal insufficiency (creatinine level $>2 \mathrm{mg} / \mathrm{dL}$ ), preoperative hemoglobin level, histology (adenocarcinoma vs squamous, vs others), induction chemotherapy, and SF36v2 PCS and MCS QoL scales. For this study, PCS and MCS were categorized according to their values higher or lower than 50 (general population norms). Survival was defined as the interval between surgery to death or last contact. Patients who were not reported as dead at the time of the analysis were censored at the date they were last known to be alive.

Survival distribution was estimated by the Kaplan-Meier method. Significant differences in probability of surviving between the strata were evaluated by log-rank test.

Cox multivariable proportional hazards regression analysis was used to evaluate the effects of prognostic factors on survival. Predictors with $P<.1$ at univariable analysis (univariable Cox regression analysis for numeric variables and log-rank test for categorical variables) were used in the multivariable model.

Only 1 variable in a set of variables with a correlation coefficient greater than 0.5 was selected (by bootstrap procedure) and used in the regression model to avoid multicollinearity.

For the purpose of constructing the aggregate score, numeric variables were tested for a threshold effect and dichotomized by using receiver operating characteristic (curve) analysis (for identifying the best cutoff).

Bootstrap bagging with 1000 samples was used to assess the stability of the multivariable regression analysis predictors. In the bootstrap analysis, 1000 samples of the same size as the original population were drawn, with replacement from the original data set. A Cox proportional hazards regression model was repeated in each of these samples. If the final model variables occurred in most $(>50 \%)$ of the bootstrap models, the original final regression model can be judged to be stable. ${ }^{9}$ Only those variables with $P<.1$ and a bootstrap frequency of greater than $50 \%$ were retained in the final model and used to construct the score.

\section{Construction of the Survival Aggregate Score}

The scoring system was developed by proportional weighing of the significant predictor estimates, assigning a value of 1 to the smallest coefficient. An aggregate risk score was generated for each patient by summing each estimate. Finally, patients were grouped in classes of incremental risk according to their total score.

The stability of the risk score across multiple populations was further tested in 1000 bootstrapped samples drawn with replacement from the original data set.

A significance level of .05 was chosen to assess the statistical significance. All tests were performed on Stata 9.0 statistical software (Stata Corporation, College Station, Tex).

\section{RESULTS}

The characteristics of the patients included in this study are shown in Table 1.

The 5-year and median overall survival levels in the entire population were $55 \%$ and 75 months, respectively.

The following variables were associated with overall survival at univariable analysis and were used as independent predictors in the Cox proportional hazards regression analysis: age $(P=.008), \mathrm{FEV}_{1}$ percentage $(P=.04), \mathrm{FEV}_{1} /$ FVC ratio $(P=.04)$, DLCO percentage $(P=.02)$, ECOG score $(P=.01)$, and PCS less than $50(P=.006)$.

To construct the aggregate score, receiver operating characteristic (curve) analysis was used to categorize the numeric variables. The following best thresholds were found: aged older than 70 years (c index, 0.58$), \mathrm{FEV}_{1}$ lower than $80 \%$ (c index, 0.55 ), $\mathrm{FEV}_{1} / \mathrm{FVC}$ less than 0.7 (c index, 0.58), DLCO less than 70\% (c index, 0.58), and ECOG score greater than 2 (c index, 0.63 ).

Cox proportional hazards regression analysis showed that the factors negatively associated with overall survival in the 
TABLE 1. Characteristics of the 245 patients included in the study

\begin{tabular}{|c|c|c|}
\hline Variables & \multicolumn{2}{|c|}{ Value } \\
\hline Age, $y$ & \multicolumn{2}{|c|}{$67(9.7)$} \\
\hline Aged $>70 y^{*}$ & \multicolumn{2}{|c|}{114 (47) } \\
\hline FEV1, \% & \multicolumn{2}{|c|}{$84.6(19.3)$} \\
\hline DLCO, $\%$ & \multicolumn{2}{|c|}{$77.3(18.8)$} \\
\hline Male sex* & \multicolumn{2}{|c|}{$198(81)$} \\
\hline Coronary artery disease* & \multicolumn{2}{|c|}{$32(13)$} \\
\hline ASA & \multicolumn{2}{|c|}{$2.3(0.5)$} \\
\hline ECOG & \multicolumn{2}{|c|}{$0.7(0.7)$} \\
\hline PCS & \multicolumn{2}{|c|}{$50.9(7.8)$} \\
\hline MCS & \multicolumn{2}{|c|}{$46.6(12.7)$} \\
\hline Pneumonectomy* & \multicolumn{2}{|c|}{$31(13)$} \\
\hline \multirow{3}{*}{ Histology* } & Adenocarcinoma & $131(53)$ \\
\hline & Squamous & $78(32)$ \\
\hline & Others & $37(15)$ \\
\hline \multirow[t]{3}{*}{ pT stage $\dagger$} & 1 & 104 \\
\hline & 2 & 123 \\
\hline & $>2$ & 18 \\
\hline \multirow[t]{3}{*}{$\mathrm{pN}$ stage $\dagger$} & 0 & 180 \\
\hline & 1 & 31 \\
\hline & 2 & 34 \\
\hline
\end{tabular}

Results are reported as means (SDs) unless otherwise specified. $F E V_{l}$, Forced expiratory volume in 1 second; $D L C O$, carbon monoxide lung diffusion capacity; $A S A$, American Society of Anesthesiologist; ECOG, Eastern Cooperative Oncology group; $P C S$, physical component score; $M C S$, mental component score. *Data are given as number (percentage). $\dagger$ Data are given as number of patients.

entire series were PCS less than 50 (coefficient, 0.55; hazard ratio $[\mathrm{HR}], 1.7 ; 95 \%$ confidence interval $[\mathrm{CI}], 1.2-2-$ $6 ; P=.008$; bootstrap frequency, $78 \%$ ), aged older than 70 years (coefficient, 0.64; HR, 1.9; 95\% CI, 1.3-2.9; $P=.002$; bootstrap frequency, $86 \%$ ), and DLCO less than $70 \%$ (coefficient, 0.55 ; HR, 1.7; 95\% CI, 1.1-2.6; $P=.01$; bootstrap frequency, $72 \%$ ). Performance of the model was as follows: log likelihood, -463.3 ; log-rank $\chi^{2}, 22.2(P=.0001)$; and goodness of fit for the inclusion of design variables based on 2 quartiles of risk, $P=.9$.

Based on their regression coefficients of similar magnitude, a weighted score was created, assigning 1 point to all factors.

Points were summed, obtaining a cumulative aggregate score for each patient ranging from 0 to 3 . For instance, a patient older than 70 years with a PCS score lower than 50 and a DLCO less than $70 \%$ would have a score of 3 .

Patients were then grouped into 4 risk classes according to their aggregate score, which was significantly associated with incremental risk of death: class survival aggregate score (SAS) 0,0 points $(\mathrm{n}=56)$; class SAS1, 1 point $(\mathrm{n}=98)$; class SAS2, 2 points $(n=75)$; and class SAS3, 3 points $(n=16)$.

Figure 1 shows the Kaplan-Meier estimates of overall survival according to different SASs (log-rank test, $P<.0001$ ). The 5-year and median overall survival levels in the 4 groups were as follows: class SAS0, $78 \%$ and median survival not reached; class SAS1, 59\% and median survival not reached; class SAS2, $42 \%$ and 47 months; and class SAS3, $14 \%$ and 15 months. The median survival is the

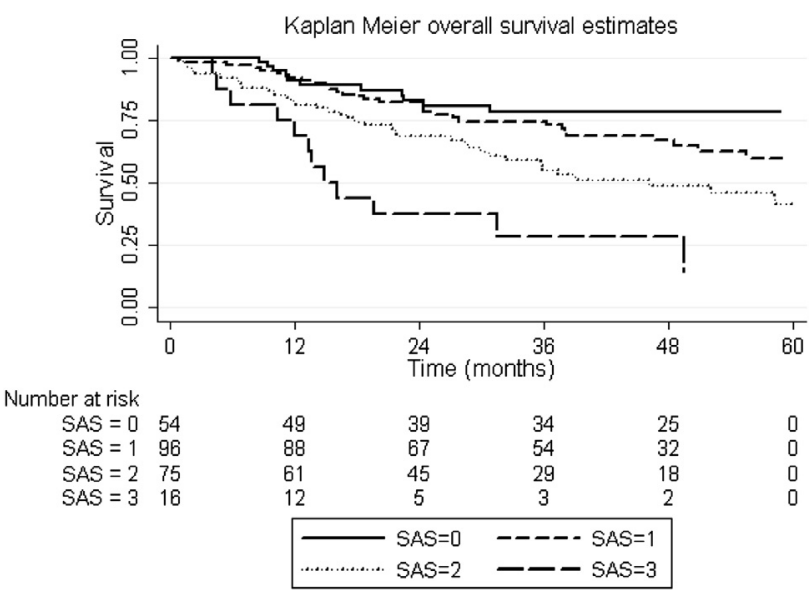

FIGURE 1. Kaplan-Meier estimates of overall survival in all patients with non-small cell lung cancer, according to different survival aggregate scores (log-rank test, $P<.0001$ ). SAS, Survival aggregate score.

time at which half the subjects have reached the event of interest. If the survival curve does not fall to $0.5(50 \%)$, then the median time cannot be computed. All log-rank pairwise comparisons between different SAS levels yielded $P<.05$.

Bootstrap bagging showed that the SAS was significantly associated with overall survival (log-rank test, $P<.05$ ) in $98 \%$ of 1000 simulated populations drawn with replacement from the original data set.

SAS was associated with overall survival in all pT stages (Table 2 and Figures E1 and E2). Limited to patients with pT1 and pT2 stages, SAS was associated with overall survival in patients with both $\mathrm{pN} 0$ and $\mathrm{pN} 1-2$ stages (Table 3 and Figures E3 and E4).

In pT1 N0 patients, the 5-year overall survival rates were $92 \%$ in SASO, $65 \%$ in SAS1, 36\% in SAS2, and $25 \%$ in SAS3 (log-rank test, $P=.003$; Figure E5).

Similarly, in pT2 N0 patients, the 5-year overall survival rates were $86 \%$ in class SAS0, $66 \%$ in class SAS1, $50 \%$ in class SAS2, and $0 \%$ in class SAS3 (log-rank test, $P=.1$ Figure E6).

Figure 2 shows the Kaplan-Meier estimates of cancerspecific survival according to SAS (log-rank test, $P<.0001)$. The 5-year and median cancer-specific survival levels in the 4 groups were as follows: class SAS0, $83 \%$ and median survival not reached; class SAS1, 71\% and median survival not reached; class SAS2, $63 \%$ and median survival not reached; and class SAS3, 17\% and 20 months.

\section{DISCUSSION}

\section{Rationale and Objective}

Patient-centered factors are increasingly reported to be associated with long-term survival in patients with cancer, ${ }^{10,11}$ including lung cancer surgical patients. ${ }^{1-6}$ Although their causal link with survival remains speculative, they can be used to refine prognostic stratification. 
TABLE 2. Median and 5-year overall survival of patients with different pT stages according to their SAS

\begin{tabular}{|c|c|c|c|c|c|c|c|c|c|}
\hline \multirow[b]{2}{*}{ pT stage } & \multicolumn{2}{|c|}{ SAS0 } & \multicolumn{2}{|c|}{ SAS1 } & \multicolumn{2}{|c|}{ SAS2 } & \multicolumn{2}{|c|}{ SAS3 } & \multirow{2}{*}{$\begin{array}{c}\text { Log-rank } \\
P \text { value }\end{array}$} \\
\hline & Median, mo & 5 y, \% & Median, mo & $5 \mathrm{y}, \%$ & Median, mo & $5 \mathrm{y}, \%$ & Median, mo & $5 \mathbf{y}, \%$ & \\
\hline 1 & $\mathrm{nr}$ & 80 & 75 & 59 & 53 & 39 & 20 & 20 & .01 \\
\hline 2 & $\mathrm{nr}$ & 82 & $\mathrm{nr}$ & 61 & 38 & 45 & 16 & 0 & .02 \\
\hline $3-4$ & 23 & 50 & $\mathrm{nr}$ & 57 & 17 & 25 & 5 & 0 & .001 \\
\hline
\end{tabular}

SAS, Survival aggregate score; $n r$, not reached.

The objective of this study was to identify objective and subjective nononcologic factors associated with survival after anatomic lung resection for NSCLC and to incorporate them into a single aggregate score.

\section{Main Finding}

An age older than 70 years at operation, a preoperative DLCO lower than $70 \%$ predicted, and a preoperative QoL PCS score lower than the norm of the general population were associated with worse overall survival. These 3 factors were used to construct a score ranging from 0 to 3 points (where 0 corresponds to 0 of the factors present and 3 corresponds to all 3 factors present). Patients with a score of 0 had a 5-year overall survival that was almost 6-fold longer than those with a score of 3 . The score remained associated with overall survival even when the patients were stratified by $\mathrm{pT}$ and $\mathrm{pN}$ stages and was also associated with cancerspecific survival.

\section{Evidence Context}

Several retrospective series have reported on the association of age and prognosis in patients with NSCLC. In general, most of the studies support that surgery of early-stage NSCLC in elderly persons is feasible and yields similar survival benefits compared with younger patients. ${ }^{12}$ Two recent analyses from the national Surveillance, Epidemiology, and End Results database found somewhat different results. In a population of approximately 15,000 patients, Owonikoko and colleagues ${ }^{13}$ found that elderly persons had worse overall survival, independent of sex, stage, and histology. However, in the surgical group, they did not observe any survival differences between elderly and younger patients. Sigel and colleagues ${ }^{14}$ found that among resected male patients, 5-year relative survival rates declined with advanced age.

In our study, an age older than 70 years significantly associated with worse overall survival. This may be partly explained by the highest incidence of competing risks in elderly patients, who are more likely to have an increased burden of comorbidities and limited life expectancy, confirming previous reports. ${ }^{15,16}$

Preoperative carbon monoxide lung diffusion capacity has previously been a predictor of overall ${ }^{1}$ and cause-specific $^{2}$ long-term survival. Ferguson and colleagues ${ }^{1}$ found that DLCO was a predictor of overall survival and that a value of $80 \%$ was the best cutoff to identify patients at increased risk of mortality. This value was similar to the cutoff found in our analysis.

There is a growing body of evidence showing the association between health-related QoL data and survival in patients with cancer. ${ }^{10}$ Although self-reported health status has been the focus of many studies in patients with advanced lung cancer stages, limited information exists about the influence of baseline QoL measures on long-term survival in patients with early-stage NSCLC who underwent surgery. Möller and Sartipy ${ }^{4}$ and Pompili and colleagues ${ }^{6}$ found that the 36-item short-form health survey (SF36) PCS score was a significant independent predictor of survival after surgery. Sloan and colleagues 5 analyzed a mixed series, including both surgical and nonsurgical patients, using the Lung Cancer Symptom Scale. They found that an overall QoL score lower than 50 was associated with poor prognosis.

In the present analysis, a PCS lower than the normal values of the general population was associated with shorter survival time after adjusting for other prognostic factors. Nevertheless, the association between self-rated health and mortality should not be inferred as a causal relationship. The PCS score may be interpreted as a parameter reflecting other unknown or unrecorded biological factors relevant to survival. ${ }^{11}$

Another hypothesis may refer to the concept of interoception, the individual's superior ability to sense and incorporate even nonconceptualized sensations of bodily status into self-ratings of health through the conscious representation of humoral and biochemical signals. ${ }^{17,18}$ This may contribute to the association of self-rated health with mortality. ${ }^{19}$

TABLE 3. Median and 5-year overall survival of patients with pT1 and pT2 stages with negative or positive lymph nodes according to their SAS

\begin{tabular}{|c|c|c|c|c|c|c|c|c|c|}
\hline \multirow[b]{2}{*}{ pN stage } & \multicolumn{2}{|c|}{ SAS0 } & \multicolumn{2}{|c|}{ SAS1 } & \multicolumn{2}{|c|}{ SAS2 } & \multicolumn{2}{|c|}{ SAS3 } & \multirow{2}{*}{$\begin{array}{c}\text { Log-rank } \\
P \text { value }\end{array}$} \\
\hline & Median, mo & $5 \mathrm{y}, \%$ & Median, mo & $5 \mathrm{y}, \%$ & Median, mo & $5 \mathrm{y}, \%$ & Median, mo & $5 \mathrm{y}, \%$ & \\
\hline 0 & $\mathrm{nr}$ & 88 & $\mathrm{nr}$ & 66 & 59 & 45 & 32 & 23 & .0005 \\
\hline $1-2$ & $\mathrm{nr}$ & 57 & $\mathrm{nr}$ & 51 & 36 & 40 & 12 & 0 & .02 \\
\hline
\end{tabular}

SAS, Survival aggregate score; $n r$, not reached. 


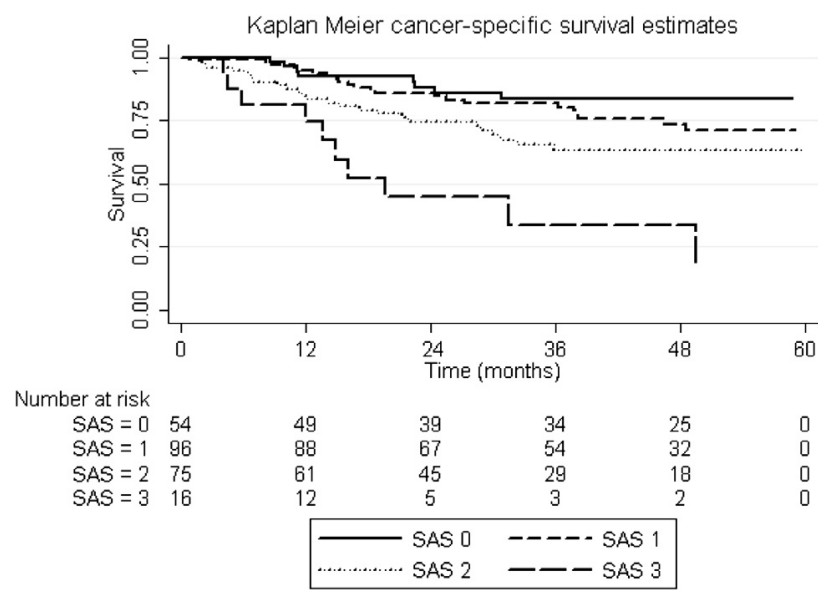

FIGURE 2. Kaplan-Meier estimates of cancer-specific survival in all patients, according to different survival aggregate scores (log-rank test, $P<.0001)$. SAS, Survival aggregate score.

\section{Caveats and Limitations}

The study included patients undergoing major anatomic pulmonary resections performed through a muscle-sparing lateral thoracotomy. The prognostic relevance of SAS in other types of resections or surgical accesses (ie, videoassisted thoracic surgery) needs to be confirmed.

We considered only preoperative variables. Perioperative changes may also have an influence on survival. ${ }^{4}$ However, postoperative changes of QoL and DLCO may reflect other treatment- and cancer-related factors, and their relationships with prognosis deserve specific investigations.

The SF36v2 scales were used to measure QoL because this was the questionnaire in use during the period of the study. More cancer-specific instruments (ie, European Organization for Research and Treatment of Cancer Quality of Life Questionnaire-30/Lung Cancer 13) may yield different results and contribute to find additional information.

Approximately $20 \%$ of the patients initially eligible for the study were lost to follow-up (LTFU). This may be, in part, explained by the absence of a centralized institutional follow-up system and the broad catchment area. We assessed the potential impact of this dropout rate on the results using 2 extreme approaches. When all LTFU patients were assumed as dead, the log-rank test for overall survival yielded a $\chi^{2}$ value of 14.3 , with $P=.002$. When all LTFU patients were assumed as alive, the log-rank test for overall survival yielded a $\chi^{2}$ value of 22.8 , with $P<.0001$. Based on this simulation, we can conclude with a certain degree of reliability that the inclusion of lost patients would have not influenced the results.

This analysis has been limited to patients who were able to complete the preoperative QoL questionnaire and to perform the preoperative exercise test. Patients unable or unwilling to do so may have specific baseline, medical, or psychosocial characteristics that deserve to be evaluated separately to verify their association with prognosis.
A larger sample size will be needed to refine and improve discrimination of the model among the highest-risk group. In fact, in our analysis, a 71-year-old patient with a DLCO of $65 \%$ and an SF36 PCS of 45 has the same SAS as an 88-year-old patient with a DLCO of $40 \%$ and an SF36 PCS of 35. This may limit the ability of this scoring system to be used as a risk-adjustment instrument in clinical trials.

\section{Clinical and Research Implications}

The discriminative ability of SAS can be used to refine prognostic stratification in patients with NSCLC who underwent lung resection for either clinical or research purposes.

Patients with a high SAS can be identified and counseled for more frequent follow-up, regardless of their pTNM stage.

Supportive pharmacologic or rehabilitative measures can be established to increase the levels of DLCO or QoL PCS score, with the objective to potentially improve prognosis.

In this regard, several studies have shown the beneficial effects of physical function on cancer recurrence mediated by a reduction in body weight and composition and changes in metabolic and sex hormones, growth factors, adipokines, immune function, or inflammation. ${ }^{20-22}$ Therefore, the implementation of preoperative rehabilitation programs capable of improving the aerobic capacity ${ }^{23-25}$ may influence residual QoL and long-term survival.

With the limitation previously mentioned, SAS can be used as a risk-adjustment instrument in clinical trials assessing the efficacy of novel surgical techniques (ie, sublobar resections in early-stage, minimally invasive thoracic surgery) or adjuvant treatments (chemotherapy or biologic agents).

Future investigations are needed to explore the feasibility and efficacy of adjuvant treatment in patients with earlystage NSCLC with a high SAS, which, as previously mentioned, may be a surrogate for a subclinical immunologic or humoral substrate favoring cancer progression. ${ }^{11,19}$

SAS, which is a patient-centered model, may be interpreted as the initial step toward the construction of a more complex model, including treatment- and cancer-related prognostic factors. This cumulative model will require a larger sample size analysis to be developed.

\section{CONCLUSIONS}

In conclusion, we developed an aggregate score incorporating patient-based preoperative objective (DLCO and age) and subjective (QoL PCS score) factors, which was associated with prognosis after lung resection for NSCLC.

Higher scores corresponding to worse patient conditions are associated with poorer overall and cancer-specific survival. 
If these results will be confirmed by independent investigations, this index may be used to refine stratification of prognosis for clinical and research purposes.

\section{References}

1. Ferguson MK, Dignam JJ, Siddique J, Vigneswaran WT, Celauro AD. Diffusing capacity predicts long-term survival after lung resection for cancer. Eur J Cardiothorac Surg. 2012;41:e81-6.

2. Liptay MJ, Basu S, Hoaglin MC, Freedman N, Faber LP, Warren WH, et al. Diffusion lung capacity for carbon monoxide (DLCO) is an independent prognostic factor for long-term survival after curative lung resection for cancer. J Surg Oncol. 2009;100:703-7.

3. Brunelli A, Pompili C, Berardi R, Mazzanti P, Onofri A, Salati M, et al. Performance at preoperative stair-climbing test is associated with prognosis after pulmonary resection in stage I non-small cell lung cancer. Ann Thorac Surg. 2012; 93:1796-800.

4. Möller A, Sartipy U. Associations between changes in quality of life and survival after lung cancer surgery. J Thorac Oncol. 2012;7:183-7.

5. Sloan JA, Zhao X, Novotny PJ, Wampfler J, Garces Y, Clark MM, et al. Relationship between deficits in overall quality of life and non-small-cell lung cancer survival. J Clin Oncol. 2012;30:1498-504.

6. Pompili C, Salati M, Refai M, Berardi R, Onofri A, Mazzanti P, et al. Preoperative quality of life predicts survival following pulmonary resection in stage I non-small-cell lung cancer. Eur J Cardiothorac Surg. 2013;43:905-10.

7. Union Internationale Contre le Cancer. TNM Classification of Malignant Tumours. 6th ed. New York, NY: Wiley-Liss; 2002:272.

8. Ware JE Jr, Sherbourn CD. The MOS 36-item short-form health survey (SF36), I: conceptual framework and item selection. Med Care. 1992;30:473-83.

9. Blackstone EH. Breaking down barriers: helpful breakthrough statistical methods you need to understand better. J Thorac Cardiovasc Surg. 2001;122: 430-9.

10. Montazeri A. Quality of life data as prognostic indicators of survival in cancer patients: an overview of the literature from 1982 to 2008. Health Qual Life Outcomes. 2009;7:102-23.

11. Jylhä M. What is self-rated health and why does it predict mortality? towards a unified conceptual model. Soc Sci Med. 2009;69:307-16.
12. Pallis AG, Fortpied C, Wedding U, Van Nes MC, Penninckx B, Ring A, et al. EORTC elderly task force position paper: approach to the older cancer patient. Eur J Cancer. 2010;46:1502-13.

13. Owonikoko TK, Ragin CC, Belani CP, Oton AB, Gooding WE, Taioli E, et al. Lung cancer in elderly patients: an analysis of the surveillance, epidemiology, and end results database. J Clin Oncol. 2007;25:5570-7.

14. Sigel K, Bonomi M, Packer S, Wisnivesky J. Effect of age on survival of clinical stage I non-small-cell lung cancer. Ann Surg Oncol. 2009;16:1912-7.

15. Chang MY, Mentzer SJ, Colson YL, Colson YL, Linden PA, Jaklitsch MT, et al. Factors predicting poor survival after resection of stage IA non-small cell lung cancer. J Thorac Cardiovasc Surg. 2007;134:850-6.

16. Asmis TR, Ding K, Seymour L, Shepherd FA, Leighl NB, Winton TL, et al. National Cancer Institute of Canada Clinical Trials Group. Age and comorbidity as independent prognostic factors in the treatment of non small-cell lung cancer: a review of National Cancer Institute of Canada Clinical Trials Group trials. J Clin Oncol. 2008;26:54-9.

17. Cameron OG. Interoception: the inside story-a model for psychosomatic processes. Psychosom Med. 2001;63:697-710.

18. Craig AD. Interoception: the sense of the physiological condition of the body. Curr Opin Neurobiol. 2003;13:500-5.

19. Littrell J. The mind-body connection: not just a theory anymore. Soc Work Health Care. 2008;46:17-37.

20. Ingram C, Courneya KS, Kingston D. The effects of exercise on body weight and composition in breast cancer survivors: an integrative systematic review. Oncol Nurs Forum. 2006;33:937-47.

21. Chlebowski RT, Aiello E, McTiernan A. Weight loss in breast cancer patient management. J Clin Oncol. 2002;20:1128-43.

22. McTiernan A, Ulrich C, Slate S, Potter J. Physical activity and cancer etiology: associations and mechanisms. Cancer Causes Control. 1998;9:487-509.

23. Nici L. Preoperative and postoperative pulmonary rehabilitation in lung cancer patients. Thorac Surg Clin. 2008;18:39-43.

24. Nagarajan K, Bennett A, Agostini P, Naidu B. Is preoperative physiotherapy/pulmonary rehabilitation beneficial in lung resection patients. Interact Cardiovasc Thorac Surg. 2011;13:300-2.

25. Jones LW, Eves ND, Peterson BL, Garst J, Crawford J, West MJ, et al. Safety and feasibility of aerobic training on cardiopulmonary function and quality of life in postsurgical non-small cell lung cancer patients: a pilot study. Cancer. 2008;113: 3430-9. 


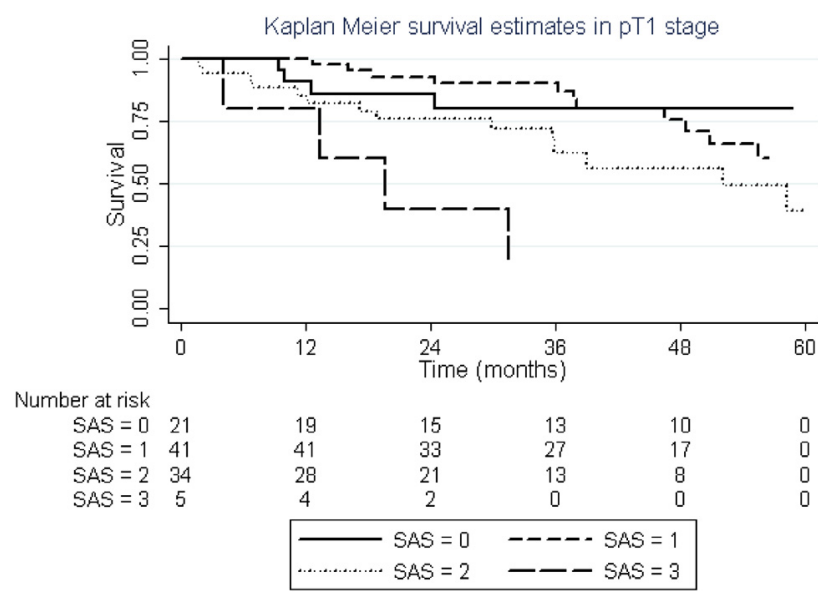

FIGURE E1. Kaplan-Meier estimates of overall survival in patients with pT1 stage non-small cell lung cancer, according to different survival aggregate scores (log-rank test, $P=.01) . S A S$, Survival aggregate score.

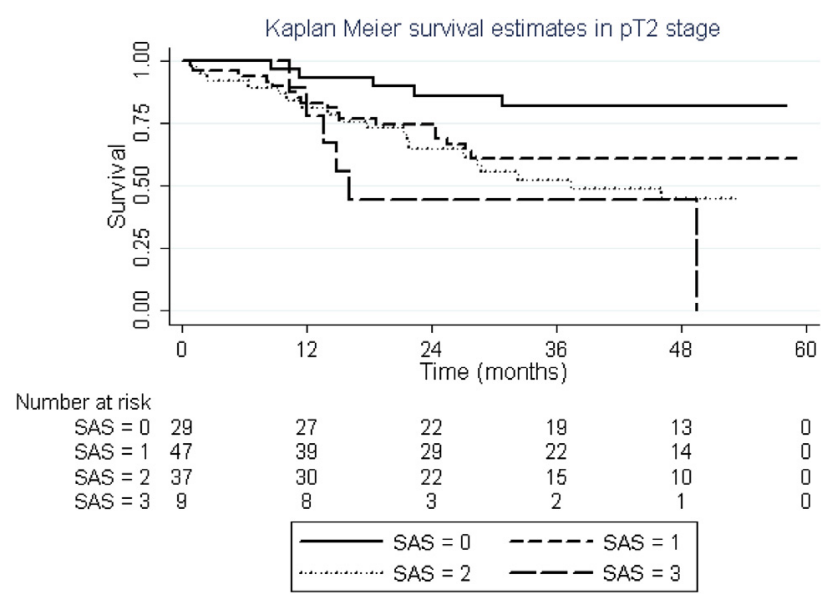

FIGURE E2. Kaplan-Meier estimates of overall survival in patients with pT2 stage non-small cell lung cancer, according to different survival aggregate scores (log-rank test, $P=.02)$. SAS, Survival aggregate score.

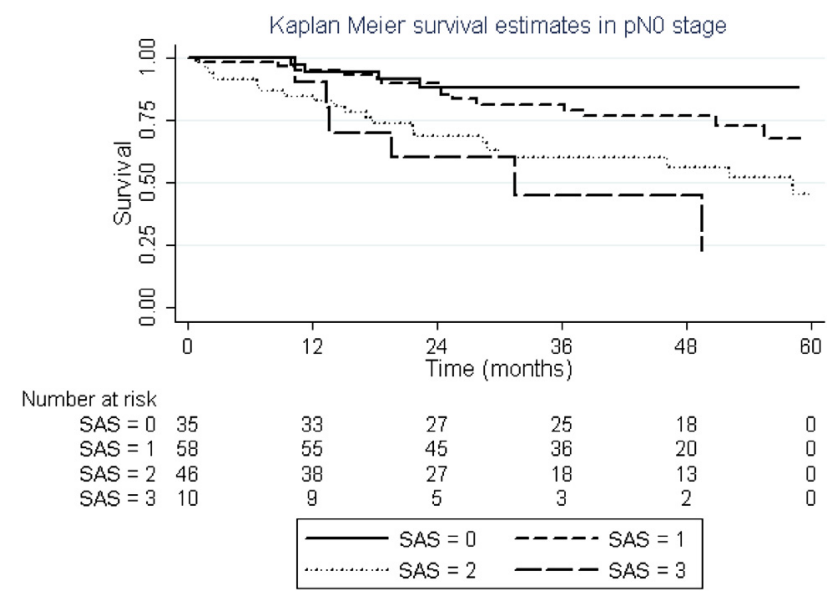

FIGURE E3. Kaplan-Meier estimates of overall survival in patients with pT1-2 N0 stage non-small cell lung cancer, according to different survival aggregate scores (log-rank test, $P=.0005)$. SAS, Survival aggregate score.

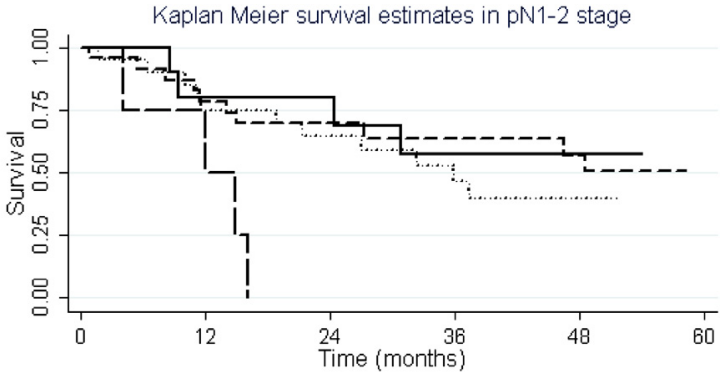

Number at risk

\begin{tabular}{|c|c|c|c|c|}
\hline$S A S=0 \quad 10$ & 8 & 7 & 5 & 3 \\
\hline$S A S=12$ & 18 & 13 & 10 & 9 \\
\hline$S A S=2$ & 15 & 11 & 7 & 4 \\
\hline$S A S=3 \quad 4$ & 3 & 0 & 0 & 0 \\
\hline & & $S A S=0$ & --1 & \\
\hline & 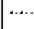 & SAS $=2$ & - - & \\
\hline
\end{tabular}

FIGURE E4. Kaplan-Meier estimates of overall survival in patients with pT1-2 N1-2 stage non-small cell lung cancer, according to different survival aggregate scores (log-rank test, $P=.02) . S A S$, Survival aggregate score. 

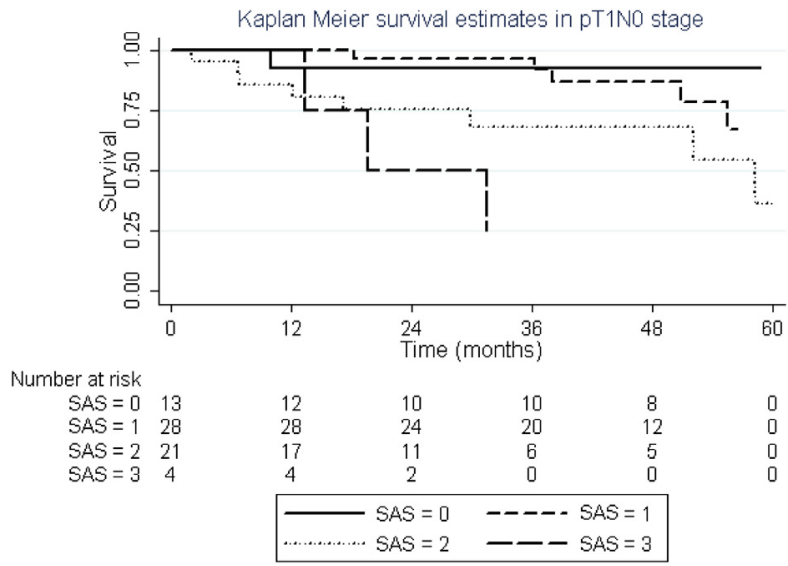

FIGURE E5. Kaplan-Meier estimates of overall survival in patients with pT1 N0 stage non-small cell lung cancer, according to different survival aggregate scores (log-rank test, $P=.04$ ). $S A S$, Survival aggregate score.

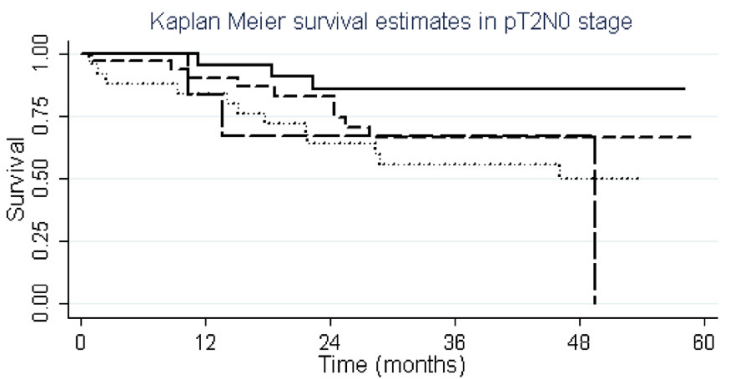

Number at risk

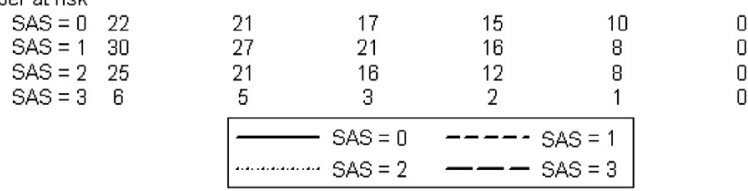

FIGURE E6. Kaplan-Meier estimates of overall survival in patients with pT2 N0 stage non-small cell lung cancer, according to different survival aggregate scores (log-rank test, $P=.1)$. SAS, Survival aggregate score. 